Methods To address our aims, we interviewed 9 patients with chronic diseases and 7 relatives. We used constant comparative analyses to categorize their key elements of ACP.

Results Patients and relatives would use search terms such as 'ACP', 'care/treatment plan', 'disease trajectory', 'advance directive' and patient associations. They would appreciate information on ACP and its importance; how to adapt ACP to individual needs; values and quality of life; diseases; treatment and care options; and communication of preferences. Furthermore, interviewees mentioned additional needs, such as how to get support, explanation of persons involved in ACP, encouragement to think about ACP, information for relatives, and peer support.

Conclusion(s) Key elements of ACP according to patients and relatives are in line with the EAPC consensus concept according to professionals. However, patients and relatives also mentioned additional elements, indicating their concept of ACP may be broader. Including the elements and search terms in ACP information may enhance online findability and may help meeting information needs.

Funding The Netherlands Organisation for Health Research and Development (ZonMw).

\section{P55 NURSES' UNDERSTANDING AND PERSPECTIVES OF ADVANCE CARE PLANNING IN COPD}

C Kelly, N Cornally*. UCC graduate, GALWAY, Ireland

\subsection{6/spcare-2019-ACPICONGRESSABS.138}

Background Advance care planning (ACP) presents as a current issue within an Irish context. Recent publications by The Irish Hospice Foundation include documents 'Think Ahead' and 'Planning for the Future with COPD'. In 2015 The Assisted Decision-Making Capacity Act was published. Currently, the HSE are in the process of publishing professional guidelines for practice in relation to ACP (HSE 2018.) Much of the existing literature relates to physician's views on ACP in COPD. Articles relating to nurse's understanding of ACP entail more towards other patient subgroups such as oncology patients and the older adult.

Method A descriptive qualitative design was used. Eight staff nurses from a respiratory unit were recruited. Semi-structured interviews were audio-recorded. A topic guide from a previous study was adapted.

Results Four categories were identified; the importance of ACP in COPD, facilitators to ACP in COPD, barriers to ACP in COPD and the role of the nurse. Each category was further subcategorised.

Conclusion Nurses identified the importance of ACP in COPD. Benefits included symptom specific factors but also an improvement in the overall quality of care patients could receive. A number of facilitating factors and challenges were identified by the sample in the provision of ACP. Nurses identified themselves as playing an important and broad role in ACP. Future implications were identified for education, research and practice. Recommendations include an increase in education across both undergraduate and postgraduate forums, further research and the appointment of lead nurses in relation to ACP in COPD patients.

\section{P56 ENHANCING THE END OF LIFE (EOL) COMMUNICATION: EVALUATION OF THE 'EOL CARE EDUCATION' FOR NURSES IN ACUTE CARE SETTING}

${ }^{1} \mathrm{M}$ Eto*, ${ }^{2} \mathrm{~K}$ Fujimoto, ${ }^{1} \mathrm{C}$ Koyama, ${ }^{1} \mathrm{~N}$ Ishikawa, ${ }^{1} \mathrm{M}$ Shimada, ${ }^{1} \mathrm{Y}$ Maehara. ${ }^{1}$ Bell-land General Hospital, Sakai, Osaka, Japan; ${ }^{2}$ Hannan City Hospital, Hannan, Japan

\subsection{6/spcare-2019-ACPICONGRESSABS.139}

Background It is critical to discuss with advance cancer patients on their EOL issues to improve the quality for their future care. Japanese nurses, however, are apt to feel uncomfortable to deal with such discussions with these patients because most of them have limited opportunities for the training for EOL communication.

We investigated whether 'EOL care education course' for nurses may help improving their knowledge and skills on EOL care, including communication ability.

Methods We provided 15 nurse participants with the educational course based on the ELNEC Core Curriculum including communication and ethical consideration. They all had more than 2 to 25 years of nursing experience. Efficacy of the course was evaluated by using the End of Life Nursing Education-Japan Core Quiz.

Results The overall correct answer rate before the course was $66 \%$ which was significantly improved to $86 \%$ after the training $(p<0.05)$. The learning effect was obtained about the knowledge and attitude towards the EOL care by taking the course. The participants felt it challenging to deal with ethical facets and patients' spiritual pains. The results, however, showed an increase in the correct answer rates on 'communication skills' from $70 \%$ to $93 \%$, before and after the course, respectively. We believe that the practical role play during the course contributed this improvement.

Conclusions The education course we provided improved knowledge and skills for EOL communication in the nurse participants. We learned that in the future the course needs more attention on the 'ethical consideration' and 'spiritual care'.

\section{P57 COLLABORATIVE ADVANCE CARE PLANNING: IMPROVING PALLIATIVE CARE BY STRUCTURED COMMUNICATIONS ABOUT MATTERS OF LIFE AND DEATH - THE STUDY PROTOCOL}

${ }^{1} \mathrm{~J} \mathrm{Koch}{ }^{*},{ }^{1} \mathrm{M}$ Koch, IJ Riera-Knorrenschild, ${ }^{2} \mathrm{~N}$ Timmesfeld, ${ }^{3} \mathrm{~N}$ Leppin, ${ }^{3} \mathrm{~K}$ Nagelschmidt, ${ }^{1} \mathrm{P}$ Von Blanckenburg. ${ }^{1}$ Philipps-University Marburg, Marburg, Germany; ${ }^{2}$ Ruhr University, Bochum, Germany; ${ }^{3}$ Philipps University, Marburg, Germany

\subsection{6/spcare-2019-ACPICONGRESSABS. 140}

Background Implementation of advance care planning (ACP) is proceeding in the western world and often recognised as a sufficient approach to ensure patients' wishes for end of life (EoL) care. There is evidence that patient related outcomes are improved. However, information about the impact of ACP on quality of life (QoL) in palliative cancer patients is missing.

Methods This randomised controlled trial investigates the efficacy and effectiveness of a collaborative ACP (cACP) intervention in palliative cancer patients and their care givers by comparing three groups: 1. cACP-Intervention; 2. Supportive intervention 3. Treatment as usual. The cACP-intervention 